\title{
Atuação do fisioterapeuta nos cuidados paliativos em pacientes adultos: revisão integrativa
}

\author{
The performance of the physiotherapist in palliative care in adult patients: Integrative \\ review \\ El papel del fisioterapeuta en los cuidados paliativos en pacientes adultos: una revisión \\ integradora
}

Vívian Maria Siqueira Machado ${ }^{1 *}$, Álissa Kinara Coimbra ${ }^{1}$, Patrick Augusto da Silva Trindade ${ }^{1}$, Pedro Ivo Carmo Campos², Poliana de Cássia Ferreira1', Rafaell Ribeiro Brasil ${ }^{1}$.

\section{RESUMO}

Objetivo: Demonstrar a atuação do fisioterapeuta nos cuidados paliativos em pacientes adultos, os principais recursos e os níveis de evidências científicos dos estudos. Métodos: Revisão integrativa realizada nas bases de dados: Biblioteca Virtual em Saúde (BVS) e Medline, nos últimos 10 anos. Os Descritores utilizados foram: "Palliative Care" e "Physical Therapists" e os Medical Subject Headins: "Physical Therapy Modalities" e "Palliative Care". Resultados: Foram obtidos 10 estudos: 70\% nível de evidência IV, $20 \%$ nível I e 10\% nível II. As atuações do fisioterapeuta destacam-se: na dor e seu aspecto cognitivo-afetivo, dispnéia, depuração de muco, fadiga, alterações linfáticas e gerenciamento. Os principais recursos foram: terapias manuais, eletroterapia, termoterapia, cinesioterapia, apoio espiritual e emocional, relaxamento, eletroestimulação, oxigenoterapia, exercícios de consciência respiratória, assistência à tosse, drenagem postural, conservação de energia, terapia complexa descongestiva, instrução e capacitação de cuidados. Considerações finais: Os principais sintomas manejados pelo fisioterapeuta no cuidado paliativo são a dor, dispnéia, secretividade, fadiga, problemas linfáticos e edema, porém algumas intervenções necessitam maior evidência de eficácia.

Palavras-chave: Cuidados paliativos, Fisioterapia, Fisioterapeuta.

\section{ABSTRACT}

Objective: To demonstrate the physiotherapist's performance in palliative care in adult patients, the main resources and the levels of scientific evidence of the studies. Methods: Integrative review performed in the databases: Virtual Health Library and Medline, in the last 10 years. The Descriptors used were: "Palliative Care" and "Physical Therapists" and the Medical Subject Headins: "Physical Therapy Modalities" and "Palliative Care". Results: 10 studies were obtained: $70 \%$ level of evidence IV, $20 \%$ level I and $10 \%$ level II. The physiotherapist's actions stand out: in pain and its cognitive-affective aspect, dyspnea, mucus clearance, fatigue, lymphatic alterations and management. The main resources were: manual therapies, electrotherapy, thermotherapy, kinesiotherapy, spiritual and emotional support, relaxation, electrostimulation, oxygen therapy, respiratory awareness exercises, cough assistance, postural drainage, energy conservation, complex decongestive therapy, instruction and care training. Final considerations: The main symptoms managed by the physiotherapist in palliative care are pain, dyspnea, secretivity, fatigue, lymphatic problems and edema, but some interventions require greater evidence of efficacy.

Keywords: Palliative care, Physical therapy, Physiotherapist.

${ }^{1}$ Centro Universitário Presidente Antônio Carlos (FUPAC), Barbacena - MG.

*E-mail: vivianmachado@unipac.br

${ }^{2}$ Faculdade de Medicina de Barbacena (FAME), Barbacena - MG. 


\section{RESUMEN}

Objetivo: Demostrar la actuación del fisioterapeuta en los cuidados paliativos en pacientes adultos, los principales recursos y los niveles de evidencia científica de los estudios. Métodos: Revisión integradora realizada en las bases de datos: Biblioteca Virtual de Salud y Medline en los últimos 10 años. Los descriptores utilizados fueron: "Cuidados paliativos" y "Fisioterapeutas" y los encabezamientos de los temas médicos: "Modalidades de terapia física" y "Cuidados paliativos". Resultados: Se obtuvieron 10 estudios: $70 \%$ nivel de evidencia IV, $20 \%$ nivel I y $10 \%$ nivel II. Destacan las acciones del fisioterapeuta: en el dolor y su aspecto cognitivo-afectivo, la disnea, la eliminación de moco, el cansancio, las alteraciones linfáticas y el manejo. Los principales recursos eran: terapias manuales, electroterapia, termoterapia, kinesioterapia, apoyo espiritual y emocional, relajación, electroestimulación, oxigenoterapia, ejercicios de conciencia respiratoria, asistencia para la tos, drenaje postural, conservación de la energía, terapia descongestiva compleja, instrucción y formación para el cuidado. Consideraciones finales: Los principales síntomas tratados por el fisioterapeuta en los cuidados paliativos son dolor, disnea, secretividad, fatiga, problemas linfáticos y edema, pero algunas intervenciones requieren una mayor evidencia de eficacia.

Palabras clave: Cuidados paliativos, Fisioterapia, Fisioterapeuta.

\section{INTRODUÇÃO}

As medidas que prolongam a vida em sua fase terminal e a incessante luta pela cura de doenças refratárias ao tratamento, vem distanciando a possibilidade de um final de vida digno e com ênfase em conforto de pacientes sem possibilidades terapêuticas e que necessitam de cuidados específicos. Neste contexto, o cuidado paliativo vem se diferenciando do tratamento curativo, por focar integralmente no indivíduo, principalmente no controle dos sintomas nas doenças sem expectativa ou sem resposta de melhora. (GOMES ALZ e OTHERO MB, 2016)

Historicamente o conceito de Cuidados Paliativos foi criado no Reino Unido pela enfermeira e médica Cicely Saunders, que o ano de 1967 formalizou a dedicação de sua vida ao alívio do sofrimento humano. Data que marcou a formação do movimento hospice, derivado do latim hospitium, que significa "hospedagem ou hospitalidade", traduzindo um sentimento acolhedor. Inicialmente direcionados para portadores de câncer em estágio avançado, estes cuidados ganharam notoriedade mundial e foram ampliados para outras doenças crônicas (GOMES ALZ, 2016).

No Brasil ainda temos poucas instituições especializadas neste tipo cuidado, se tratando de oito hospices e 131 hospitais com serviço especializado até 2018. Apesar da excelência de atendimento, ainda são serviços isolados e o sistema de saúde brasileiro está atrasado na integração e apoio adequado a estes serviços (SILVA NVC, 2019).

O pensamento dos cuidados paliativos busca o alívio da dor e demais sintomas angustiantes, não acelerando e nem adiando a morte, promovendo ao paciente viver bem enquanto possível, integrando os aspectos psicológico, sociais e espirituais no seu cuidado, além de disponibilizar apoio aos familiares durante o período de luto (NASCIMENTO DM, et al., 2013).

Para atender a complexidade do cuidado paliativo de pacientes e dos familiares ao longo da evolução da doença a Organização Mundial de Saúde (OMS) definiu a necessidade da atuação de equipes multiprofissionais (FERNANDO G e HUGHES S,2019).

A assistência provida por uma equipe multidisciplinar é fundamental, pois adota uma abordagem humanista e integrada, demonstrando que nenhuma profissão consegue abranger todos os aspectos envolvidos no tratamento de pacientes sob esses cuidados. É fundamental o trabalho coletivo, pois permite a sinergia de habilidades visando promover uma assistência completa nos aspectos físico, mental, social e espiritual (FERNANDO G e HUGHES S, 2019).

Fatores ambientais como doenças adquiridas e traumas, ou fatores genéticos podem levar a distúrbios funcionais do movimento e por consequência interferir na autonomia e qualidade de vida dos indivíduos. 
Dentre as ciências da saúde, cabe à fisioterapia prevenir e tratar distúrbios funcionais dos movimentos e suas repercussões orgânicas e sistêmicas no corpo humano, podendo assim restaurar, manter ou desenvolver a funcionalidade e assim melhorar a qualidade de vida do paciente (CREFITO 4, 2015).

Em uma linha de cuidados de saúde, diversas são as funções atribuídas ao fisioterapeuta, tornado-se responsável pela elaboração do diagnóstico cinético-funcional, prescrição, planejamento, organização, análise, supervisão e avaliação de projetos fisioterapêuticos. Também é responsável por averiguar a eficácia de sua abordagem, resolutividade e as condições de alta do paciente submetido a estas práticas de saúde (WILSON CM, et al., 2017).

Dentro do cuidado paliativo, sua atuação é de forma complementar, traçando um plano de assistência e auxiliando o paciente a se desenvolver de forma ativa, adaptando-o ao desgaste físico e repercussões emocionais, sociais e espirituais conforme o percurso da enfermidade até sua morte, com a finalidade de manter, preservar, aumentar ou restaurar a integridade de órgãos, sistemas ou função (WILSON CM, et al., 2017).

Ressalta-se ainda carência de literatura nacional sobre o tema, justificando a relevância deste estudo, contribuindo na produção de novas evidências científicas sobre a atuação do fisioterapeuta nos cuidados paliativos em adultos, bem como, para o conhecimento entre os profissionais da saúde e de toda sociedade. Portanto, o objetivo deste trabalho é demonstrar qual à atuação do fisioterapeuta e os principais recursos no manejo de pacientes adultos em cuidados paliativos, além de apresentar os níveis de evidências científicos dos estudos selecionados.

\section{MÉTODOS}

Este estudo trata-se de uma revisão integrativa da literatura científica. Para sua construção foi necessário desenvolver seis etapas: Identificação do tema e definição da questão de pesquisa; estabelecimento dos critérios de inclusão e exclusão; identificação dos estudos pré-selecionados e selecionados; categorização dos estudos selecionados; análise e interpretação dos resultados e apresentação da revisão/síntese do conhecimento (EVANGELISTA CB et al., 2016).

A pergunta norteadora de pesquisa do estudo foi: Qual a atuação do fisioterapeuta nos cuidados paliativos em pacientes adultos? Utilizamos à estratégia PICO para estruturação desta questão tendo: $(\mathrm{P})$ pacientes adultos, (I) cuidados paliativos, (C) controle não se aplica, (O) outcome/desfecho descrição da atuação do fisioterapeuta nos cuidados paliativos (RIBEIRO O, et al., 2016).

Foram utilizadas as bases de dados BVS, e a base MEDLINE. As buscas foram realizadas ao longo do mês de maio do ano de 2020 por um pesquisador e os estudos avaliados por dois revisores independentes, no caso de discordância um terceiro avaliador realizou arbitragem final.

Para seleção da amostra adotamos como critérios de inclusão: Publicações disponíveis online, acesso gratuito, na íntegra, nos idiomas inglês, espanhol ou português. Foi inicialmente limitada há cinco anos, mas devido à escassez de literatura relevante, a pesquisa foi estendida para dez anos, no período de Janeiro de 2010 a Dezembro de 2019 e estudos que abordassem em seu título ou resumo as palavras fisioterapia e cuidados paliativos. Foram excluídos neste trabalho: Estudos em pediatria ou que abordassem menores de 18 anos, estudos duplicados nas bases de dados e estudos que não responderam à pergunta norteadora.

Foram utilizados os Descritores em Ciências da Saúde (DeCS), vinculada à BVS, e os termos medical subject headins (MeSH), vinculados ao PubMed, mecanismo de busca da base MEDLINE.Para pesquisa na BVS foram empregados os descritores exatos em inglês "Palliative Care" e "Physical Therapists"cruzados pelo operador booleano "AND". Já no PubMed para a busca dos termos MeSH foram utilizadas as palavras em inglês "Palliative Care"e "Physical Therapists", não sendo recuperado nenhum resultado. Optou-se então por utilizar o MeSH "Physical Therapy Modalities", o mesmo foi cruzado com o MeSH "Palliative Care" utilizando o operador booleano "AND". 
Foi recuperado um total de 594 estudos, após aplicação dos filtros restaram apenas 89, sendo estes analisados os títulos e resumos restando apenas 38 estudos. Após aplicação dos critérios de elegibilidade e exclusão restaram apenas 10 estudos, os quais foram lidos na íntegra e selecionados (Figura1).

Figura 1 - Fluxograma da seleção dos artigos adaptado do modelo PRISMA.
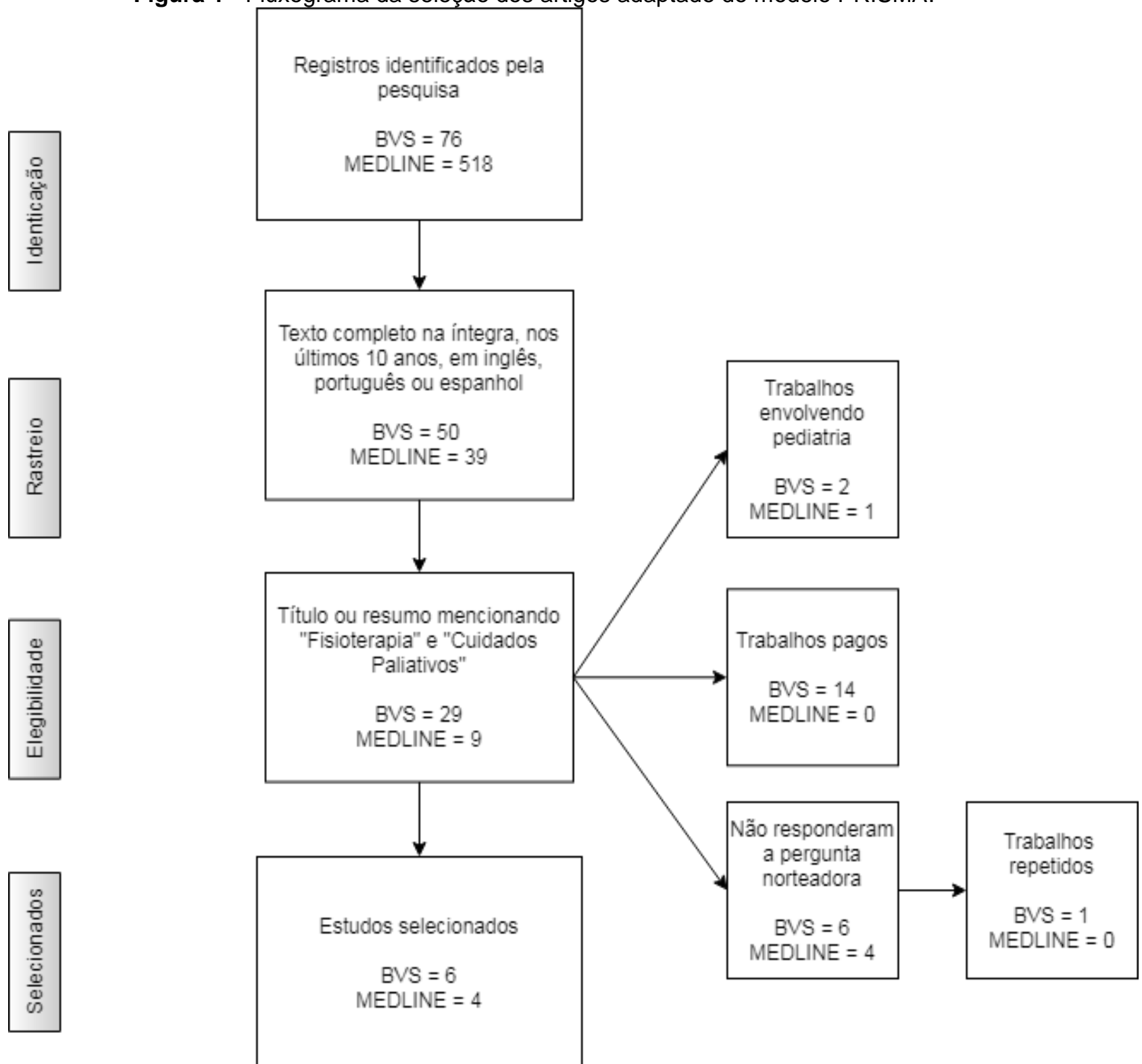

Fonte: Coimbra AK, et al., 2021.

A avaliação dos estudos quanto ao nível de evidência seguiu a Levels of Evidence for Supportive and Palliative Care Studies ( $P D Q \Theta)$, ferramenta que inclui a denominação de níveis de evidência, contribuindo na aplicabilidade de intervenções ou abordagens específicas. Os níveis de evidências são determinados por cada Conselho Editorial do PDQ, composta por especialistas em cuidados do câncer além de outras especialidades.

\section{RESULTADOS}

\section{Categorização dos estudos selecionados}

Após realização da busca nas bases de dados, 10 estudos foram selecionados conforme os critérios de seleção propostos nesta revisão integrativa. Os mesmos foram identificados de A1 a A10 (Quadro 1). 
Quadro 1 - Identificação e descrição dos estudos, autor, ano, país, periódico, fator de impacto e objetivo.

\begin{tabular}{|c|c|c|c|c|c|}
\hline $\mathbf{N}^{\circ}$ & Autores e ano & País & Periódico & $\begin{array}{l}\text { Fator de } \\
\text { impacto } \\
2019-2020\end{array}$ & Objetivo do estudo \\
\hline $\mathrm{A} 1$ & PYSZORA A, et al, 2017. & Polônia & Supportive Care in Cancer & 2.860 & $\begin{array}{l}\text { Avaliar o efeito de um programa de fisioterapia na fadiga } \\
\text { relacionada ao câncer e outros sintomas em pacientes com câncer } \\
\text { avançado }\end{array}$ \\
\hline $\mathrm{A} 2$ & ARCURI JF, et al., 2016 & Brasil & BMC Palliative Care & 3.120 & $\begin{array}{l}\text { Identificar intervenções para controlar a secreção respiratória em } \\
\text { pacientes com doença crônica com mau prognóstico e verificar os } \\
\text { resultados relevantes para os cuidados paliativos. }\end{array}$ \\
\hline A3 & $\begin{array}{l}\text { OLSSON MÖLLER, U, et al., } \\
2018\end{array}$ & Suécia & BMC Palliative Care & 3.120 & $\begin{array}{l}\text { Explorar a variedade de atividades descritas pelos fisioterapeutas } \\
\text { no atendimento das necessidades e problemas dos pacientes e de } \\
\text { suas famílias em ambientes de cuidados paliativos especializados }\end{array}$ \\
\hline A4 & $\begin{array}{l}\text { BAUSEWEIN C, SIMON ST, } \\
2013\end{array}$ & Alemanha & Deutsches Ärzteblatt International & 1.190 & $\begin{array}{l}\text { Familiarizar o leitor com a necessidade de avaliação padronizada } \\
\text { da falta de ar e tosse em pacientes com doença avançada, opções } \\
\text { de tratamentos não farmacológicos, medicamentos mais } \\
\text { importantes e o papel do uso adequado e criterioso do oxigênio em } \\
\text { seu tratamento. }\end{array}$ \\
\hline A5 & CHIGBO N, et al., 2015 & Nigeria & Nigerian Journal of Clinical Practice & 0.480 & $\begin{array}{l}\text { Explorar os problemas éticos enfrentados pelos fisioterapeutas para } \\
\text { proverem intervenções para pacientes portadores de doença } \\
\text { terminal no contexto Africano. }\end{array}$ \\
\hline A6 & $\begin{array}{l}\text { OLIVEIRA DE T, BOMBARDA } \\
\text { TB, MORIGUCHI CS, } 2019\end{array}$ & Brasil & Cadernos Saúde Coletiva & 1.000 & $\begin{array}{l}\text { Tecer reflexões acerca da atuação do fisioterapeuta em cuidados } \\
\text { paliativos no contexto da atenção primária em saúde, a partir de } \\
\text { fundamentos, princípios e diretrizes que sustentam esse cuidado. }\end{array}$ \\
\hline A7 & $\begin{array}{c}\text { MCLEOD KE, NORMAN KE, } \\
2019\end{array}$ & Canadá & $\begin{array}{c}\text { Physiothe-rapy Research } \\
\text { International }\end{array}$ & 1.330 & $\begin{array}{l}\text { Descrever idéias de entrevistados sobre a experiência dos } \\
\text { fisioterapeutas que prestam cuidados paliativos em Ontário e suas } \\
\text { percepções sobre o papel e o valor do envolvimento dos } \\
\text { fisioterapeutas nos cuidados paliativos. }\end{array}$ \\
\hline A8 & KUMAR SP, SAHA S, 2011 & Índia & Indian Journal of Palliative Care & 1.000 & $\begin{array}{l}\text { Atualizar os fisioterapeutas que trabalham em cuidados paliativos, } \\
\text { sobre a classificação da dor baseada em mecanismos e sua } \\
\text { interpretação, com evidências terapêuticas disponíveis para } \\
\text { fornecer o melhor atendimento ao paciente usando fisioterapia. }\end{array}$ \\
\hline A9 & KUMAR SP, JIM A, 2010 & Índia & Indian Journal of Palliative Care & 1.000 & $\begin{array}{l}\text { Atualizar os médicos de cuidados paliativos, fisioterapeutas e outros } \\
\text { membros da equipe sobre o papel do fisioterapeuta em uma equipe } \\
\text { de cuidados paliativos, com uma visão detalhada dos métodos de } \\
\text { tratamento de fisioterapia e suas evidências para aplicação em } \\
\text { condições que requerem cuidados paliativos. }\end{array}$ \\
\hline A10 & FARIA L, 2010 & Brasil & $\begin{array}{l}\text { História, Ciências, Saúde } \\
\text { Manguinhos }\end{array}$ & 0.320 & Não apresentou objetivo \\
\hline
\end{tabular}

Fonte: Coimbra AK, et al., 2021 
De acordo com os dados apresentados, foi publicado em média um artigo por ano (Quadro 2). Ressaltase que nos anos de 2012 e 2014 não houve publicações, já em 2010 e 2019 foram publicados dois estudos respectivamente. Salientamos que dos 10 artigos selecionados, $80 \%$ foram desenvolvidos internacionalmente e no idioma da língua inglesa, sendo apenas $20 \%$ encontrados em periódicos brasileiros.

Observa-se também que as publicações dos estudos apresentaram-se em oito periódicos diferentes, com predomínio em dois destes, ambas específicas de cuidados paliativos, o Indian Journal of Palliative Care que possui fator impacto 1.000 e a BMC Palliative Carecom fator de impacto 3.120, tendo este maior média de citações de artigos publicados.

\section{Apresentação dos resultados quanto ao nível de evidência}

A avaliação dos estudos quanto ao nível de evidência seguiu a Levels of Evidence for Supportive and Palliative Care Studies (PDQ®).

Quadro 2 - Nível de evidência dos estudos baseado no Levels of Evidence for Supportive and Palliative Care Studies (PDQ®).

\begin{tabular}{|c|c|c|}
\hline $\mathbf{N}^{\circ}$ & Tipo de estudo & $\begin{array}{c}\text { Nível de } \\
\text { evidência } P D Q \circledR\end{array}$ \\
\hline A1 & Ensaio Clínico Controlado Randomizado & Nível I \\
\hline A2 & Revisão sistemática com ensaios clínicos randomizados e observacionais & Nível I \\
\hline A3 & Descritivo com abordagem qualitativa. Série de casos & Nível II \\
\hline A4 & Revisão narrativa & Nível IV \\
\hline A5 & Revisão narrativa & Nível IV \\
\hline A6 & Ensaio teórico de reflexão & Nível IV \\
\hline A7 & Série de opiniões de especialistas. & Nível IV \\
\hline A8 & Opinião de especialista. & Nível IV \\
\hline A9 & Opinião de especialista & Nível IV \\
\hline A10 & Opinião de especialista & Nível IV \\
\hline
\end{tabular}

Fonte: Coimbra AK, et al., 2021; dados extraídos no Levels of Evidence for Supportive and Palliative Care Studies $(P D Q \mathbb{R})$.

Em relação aos níveis de evidências obteve-se 20\% dos estudos com nível I de evidência, 10\% nível II e $70 \%$ com nível IV de evidência, mesmo utilizando-se de ferramenta específica para avaliação de nível de evidência em estudos de cuidado paliativo. Diante deste panorama, notamos que a maior parte dos estudos foi desenvolvida em uma abordagem em opiniões de especialistas e revisões narrativas.

\section{Apresentação das sínteses de resultados}

A seguir está apresentada a síntese dos resultados das principais atuações e recursos utilizados pelo fisioterapeuta no manejo do cuidado paliativo em adulto dos 10 estudos selecionados (Quadro 3). 
Quadro 3 - Atuação e recursos disponíveis ao fisioterapeuta no manejo das condições apresentadas em cuidados paliativos pelos estudos obtidos.

\begin{tabular}{|c|c|}
\hline$x^{2}$ & ARTIGOS EM QUE É CITADO \\
\hline Atuação na dor & Artigos \\
\hline Terapias manuais & $\mathrm{A} 1, \mathrm{~A} 6, \mathrm{~A} 8, \mathrm{~A} 9$ e $\mathrm{A} 10$ \\
\hline Eletroterapia & $\mathrm{A} 3, \mathrm{~A} 6, \mathrm{~A} 8, \mathrm{~A} 9$ e $\mathrm{A} 10$ \\
\hline Termoterapia & $\mathrm{A} 5, \mathrm{A6}, \mathrm{A} 8, \mathrm{~A} 9$ e $\mathrm{A} 10$ \\
\hline Cinesioterapia & $\mathrm{A} 6, \mathrm{~A} 8, \mathrm{~A} 9$ e $\mathrm{A} 10$ \\
\hline Exercícios aeróbicos de baixa intensidade & $\mathrm{A} 8$ \\
\hline Dessensiblização periférica & A8 \\
\hline Terapia do espelho & A8 \\
\hline Atuação no aspecto cognitivo-afetivo da dor & Artigos \\
\hline Apoio espiritual e emocional & A5 e A8 \\
\hline Técnicas para relaxamento, & $\mathrm{A} 6$ e $\mathrm{A} 8$ \\
\hline Educação do pacientes quanto à dor & A8 e A9 \\
\hline Exercícios ativos de membros & $\mathrm{A} 1$ \\
\hline Terapias manuais & $\mathrm{A} 1$ \\
\hline Mentalização do movimento indolor & A8 \\
\hline Realidade virtual & A8 \\
\hline Recursos de biofeedback & A8 \\
\hline Recursos de distração & A8 \\
\hline Técnicas de exposição & A8 \\
\hline Atuação na dispnéia & Artigos \\
\hline Eletroestimulação de membros inferiores e abdome & $\mathrm{A} 2, \mathrm{~A} 4$ e A9 \\
\hline Oxigenoterapia & A4 e A6 \\
\hline Exercícios de consciência respiratória & A6 e A9 \\
\hline Ventilador de mesa ou mão & A4 \\
\hline Ventilação não invasiva & A6 \\
\hline Ventilação mecânica invasiva & A6 \\
\hline Andador & A4 \\
\hline Caminhada e subida de escadas & A9 \\
\hline Ergometria & A9 \\
\hline Exercícios de baixa intensidade & A9 \\
\hline Expiração forçada & A9 \\
\hline Técnicas de relaxamento & A9 \\
\hline Treinamento intervalado & A9 \\
\hline Treinamento muscular respiratório & A9 \\
\hline Treinamento resistido de membros & A9 \\
\hline Atuação na depuração de muco & Artigos \\
\hline Assistência tosse & $\mathrm{A} 2, \mathrm{~A} 3$ e $\mathrm{A} 8$ \\
\hline Drenagem postural & A2 e A9 \\
\hline Ventilação percussiva & A6 e A9 \\
\hline Higiene brônquica & $\mathrm{A} 2$ \\
\hline Colete vibratorio & A6 \\
\hline Aspiração de vias aéreas & $\mathrm{A} 2$ \\
\hline Técnica de expiração forçada & A9 \\
\hline Vibração das vias aéreas & A9 \\
\hline Atuação na fadiga & Artigos \\
\hline Técnicas de conservação de energia & A5, A6 e A9 \\
\hline Exercícios ativos de membros & $\mathrm{A} 1$ \\
\hline Prevenção de estressores físicos & A5 \\
\hline Terapias manuais & $\mathrm{A} 1$ \\
\hline Atuação nas alterações linfáticas e edema & Artigos \\
\hline Fisioterapia complexa descongestiva & $\mathrm{A} 3, \mathrm{~A} 5, \mathrm{~A} 6, \mathrm{~A} 9$ e $\mathrm{A} 10$ \\
\hline Eletroterapia & A6 \\
\hline Aparelhos de compressão pneumática & A6 \\
\hline Mobilização passiva e ativa & A6 \\
\hline Atuação no gerenciamentodo cuidado paliativo & Artigos \\
\hline Instruir e capacitar cuidadores e equipe & $\mathrm{A} 3, \mathrm{~A} 5, \mathrm{~A} 7$ e A9 \\
\hline Gerenciar expectativa familiar frente ao cuidado & A5 \\
\hline Manejo e prevenção de lesões por pressão & A6 \\
\hline
\end{tabular}

Fonte: Coimbra AK, et al., 2021. 
Observa-se que as principais atuações do fisioterapeuta identificadas nos estudos se referem à dor, aspecto cognitivo-afetivo da dor, dispnéia, na depuração de muco, fadiga, nas alterações linfáticas e edema, por fim no gerenciamento do cuidado paliativo. Já os principais recursos para o manejo nestas condições foram às terapias manuais, eletroterapia, termoterapia, cinesioterapia, apoio espiritual e emocional, técnicas para relaxamento, educação do paciente quanto a dor, eletroestimulação de membros inferiores e abdômen, oxigenoterapia, exercícios de consciência respiratória, máscara de pressão expiratória positiva, assistência a tosse, drenagem postural, técnicas de conservação de energia, fisioterapia descongestiva, instruir e capacitar cuidadores e equipes.

\section{DISCUSSÃO}

Na temática sobre cuidados paliativos a análise dos números de publicações relacionada à qualidade metodológica demonstra que a maior parte dos estudos mostrou-se com baixo nível de evidência, o que nos faz acreditar insuficientes para tomadas de decisões na prática baseada em evidências do fisioterapeuta e dos profissionais da área da saúde. Outro fator é a pouca abordagem em publicações nacionais, isso pode ser demonstrado pelo reflexo da baixa adesão ao cuidado paliativo no Brasil, que ocupa a 64a colocação na capacidade de prover cuidado paliativo e na $51^{\text {a }}$ colocação na qualidade deste cuidado dentre 80 países (THE 2015 QUALITY OF DEATH INDEX, 2015). No que tange a atuação do fisioterapeuta, a discussão do cuidado paliativo sempre traz como principais escopos a dor conseguinte da dispnéia e fadiga, porém observa-se que a atuação pode abranger várias condições como (WITTRY SA, et al., 2018).

\section{Atuação do fisioterapeuta na dor}

A dor é considerada genuinamente subjetiva e pessoal, podendo estar associada a dano real ou potencial nos tecidos, sendo descrita em termos desses danos ou tanto quanto por ambas as características. Sua percepção é multidimensional, podendo se diversificar na intensidade sensorial e na qualidade, sendo também afetada por aspectos variáveis afetivo-emocional. É um dos sintomas mais perturbadores estando presente entre $70 \%$ a $90 \%$ dos pacientes em cuidados paliativos (SHOLJAKOVA M, et al., 2018). A falta de efetividade em seu controle toca todos os aspectos da qualidade de vida desses pacientes, tornando seu alívio um papel de destaque (MCGUIRE DB, et al., 2016).

Neste contexto a fisioterapia possui um grande arsenal de técnicas na complementação ao tratamento da dor. O recurso mais utilizado para o tratamento da dor em pacientes paliativos são as terapias manuais e suas variadas técnicas (ZIANI MM, et al., 2017). Dentre essas a liberação miofascial e massoterapia se destacam por possuir forte evidência científica, reduzindo a necessidade de analgesia de resgate e a ansiedade, além de melhorarem a qualidade de vida desses pacientes (CHAPMAN E, et al., 2020).

Outro recurso de destaque foi à eletrofototermoterapia sendo a eletroestimulação transcutânea (TENS), a mais citada nos artigos obtidos. Um ensaio clínico randomizado conduzido por Siemens W, et al. (2020) não evidenciou superioridade do TENS em relação ao TENS placebo, visto que ambos grupos estudados relataram alívio da dor com sua aplicação. Para Gibson W, et al. (2019) foi difícil elucidar os potenciais riscos e benefícios do uso do TENS, além de sua relação com o controle da dor e qualidade de vida.

Em contrapartida,Nakano J, et al. (2020) demonstrou alívio da média diária de dor e em até $1 \mathrm{~h}$ após o uso do TENS, sem necessidade de progressão da dose de opiódes. Como há controversa para esta e outras técnicas da eletrofototermoterapia, se fazem necessários mais estudos. O recurso de evidência científica mais sólida no alívio da dor em pacientes paliativos é a cinesioterapia e suas diversas técnicas. Sua efetividade pode ser explicada pela liberação de seretonina e opióides endógenos que diminuem o quadro álgico, além de reestabelecer a movimentação articular e tissular fisiológica após sua aplicação (GRECO FP, et al., 2019).

\section{Atuação no aspecto cognitivo-afetivo da dor}

O manejo da dor é um desafio no cuidado paliativo, isso porque a dor é subjetiva e pessoal abordada pelos aspectos físico, psíquico, social e espiritual. Quando esses aspectos são abrangidos, obtemos o conceito criado de dor total. O fisioterapeuta auxilia no apoio espiritual e emocional com intervenções de 
educação quanto à dor e técnicas de relaxamento. Neste cenário é de suma importância a abordagem multidisciplinar devido sua alta complexidade, implicando na demonstração que nenhuma profissão consegue abranger todos os aspectos relacionados à dor, permitindo a sinergia de habilidades e promovendo uma assistência completa (SILVA LFA, et al., 2017).

\section{Atuação na dispnéia}

Com o avanço da doença terminal, a dispnéia pode estar presente tornando-se um dos sintomas mais incapacitantes ao paciente. Um dos recursos mais ressaltados para este sintoma é a eletroestimulação neuromuscular, aplicada a membros inferiores e a estimulação elétrica funcional na musculatura abdominal. Justifica-se sua aplicação por otimizarem a funcionalidade, aliviarem a dispnéia, aumentarem a massa muscular, melhorarem a resistência e a tolerância aos esforços diários (KONDO T, et al., 2019).

A oxigenoterapia também é um recurso bastante utilizado na dispnéia, porém sua prescrição somente é indicada em pacientes hipoxêmicos (TORRAS JJ e PORTA-SALES J,2015). Recursos mais simples e eficazes no seu alívio,como o ventilador de mão ou de mesa, podem ser utilizados inicialmente. Seu efeito é explicado pela estimulação do nervo trigêmeo quando direcionado para o rosto do paciente, levando a redução da percepção de dispnéia pelo córtex somatossensorial (KAKO J, et al., 2020).

Caso esses pacientes apresentem menor limitação funcional, podem ser introduzidos programas de treinamento físico, incluindo caminhada e subidas de escadas, exercícios de resistência para os membros, treinamentos de força geral, treinamentos de musculatura respiratória e aeróbicos de baixa intensidade (GRANGER CL, 2016). No agravamento da dispneia pode ser empregada a ventilação mecânica não invasiva, que possui menor morbidade em relação à invasiva, reduzindo a duração da hospitalização e mortalidade, sendo opção em pacientes com ordem de não intubação e não reanimação (DIAZ DE TERAN T, et al., 2020).

\section{Atuação do fisioterapia na depuração demuco}

Pacientes em cuidados paliativos podem apresentar problemas no processo de remoção de secreções das vias aéreas devida a fatores como disfagia, doença pulmonar, piora do nível de consciência e tosse ineficaz, sendo um achado comum no processo de terminalidade (BOLAND JW e BOLAND EG, 2019).

Um recurso que vem ganhando força na facilitação da remoção de muco em pacientes colaborativos é a máscara com pressão expiratória positiva. Apesar de pouco estudada especificamente nestes pacientes outros autores discutem a efetividade de seu uso em patologias que podem evoluir com a indicação de cuidados paliativos (MCLLWAINE M, et al., 2019). Em pacientes secretivos com tosse ineficaz a tosse manualmente assistida ou máquina de tosse, se tornam preferenciais à aspiração devida maior conforto $\mathrm{e}$ menor risco de estimulação vagal (MATILDE INE, et al., 2018).

\section{Atuação do fisioterapeuta na fadiga}

Dentre os inúmeros sintomas que levam ao sofrimento dos pacientes portadores de doenças terminais, a fadiga merece atenção especial. Definida por sensação subjetiva de fraqueza, cansaço e perda de energia, pode estar diretamente relacionada na redução da qualidade de vida, da funcionalidade e interferir na eficácia do tratamento em cuidados paliativos (POORT H, et al., 2020).

Destacam-se técnicas de conservação de energia para controle inicial da fadiga, utilizando recursos como ergonomia e biomecânica, organização das atividades para redução do gasto energético e uso de órteses quando indicadas. Seus resultados levam não somente à conservação de energia como ao controle dos componentes cognitivo-afetivos da fadiga. Sua associação com exercícios é fortemente recomendada em diretrizes, sendo discutidas em diversos estudos, porém necessitando de mais estudos para definição de protocolos (POORT H, et al., 2020).

A prática de atividade física é extremamente consolidada na literatura no manejo da fadiga (BERGER FRIKKEL J, et al., 2020). Seus efeitos levam a uma série de alterações metabólicas com efeitos antiinflamatórios que potencializam a tolerância a tratamentos farmacológicos e minimizam a sarcopenia 
em condições terminais. Estudos descrevem que a prática precoce de exercícios físicos possui maior eficácia e reduz a necessidade da abordagem cognitiva complementar para a redução da fadiga (FRIKKEL J, et al., 2020; MUSTIAN KM, et al., 2017).

\section{Atuação do fisioterapeuta nas alterações linfáticas}

O edema pode levar a dor e fragilidade em pacientes em final de vida favorecido por fatores como: imobilidade, hipoproteinemia, obstrução linfática e aumento da pressão hidrostática. A primeira linha terapetêutica para o linfedema é a terapia descongestiva complexa que envolve drenagem linfática manual, cuidados com a pele, compressão com faixas e elevação do membro, sendo recomendada em vários tipos de edema. A abordagem adequada é fundamental para reconhecer as limitações terapêuticas evitando lesões ou tratamento obstinados, sendo a manutenção funcional da pele e conforto do paciente os principais objetivos (GRADALSKI T, 2019).

\section{Atuação do fisioterapeuta no gerenciamento do cuidado paliativo}

Considerando a complexidade do cuidado paliativo, a atuação do fisioterapeuta pode envolver a liderança das equipes multiprofissionais. São responsabilidades demandadas: gerenciar o plano de cuidados, o tratamento, avaliar a necessidade de referenciar para outros membros da equipe ou servir de referência quando necessário, manejar a relação profissional com paciente e cuidadores, saber abordar expectativas de desfechos, ponderar o equilíbrio entre reabilitação e suporte paliativo (BAUSEWEIN C, 2018).

Também deve instruir os pacientes e familiares: cuidados preventivos informando sobre o declínio progressivo esperado, auxiliando na aquisição de equipamentos, capacitando no posicionamento, ergonomia e transferência segura do paciente (WILSON CM, et al., 2017). Por fim a liderança do fisioterapeuta destaca-se em seu papel de referencial acadêmico, participando de pesquisas, desenvolvimento e divulgação científica e social do cuidado paliativo (BAUSEWEIN C, 2018).

\section{CONSIDERAÇÕES FINAIS}

O princípio da não maleficência deve motivar os profissionais de saúde a garantir a proteção dos pacientes terminais frente à possíveis tratamentos obstinados. A assistência do fisioterapeuta nos pacientes adultos em cuidados paliativos se destaca nos principais sintomas como a dor, dispnéia, secretividade, fadiga, problemas linfáticos e edema, além de poder gerenciar estes cuidados. $O$ arsenal de recursos disponíveis para intervenções é amplo, porém alguns destes necessitam maior evidência comprovando indicação e eficácia evitando a exposição dos pacientes à possibilidade de falha em seu manejo sintomático e risco de sofrimento em seus momentos finais.

\section{REFERÊNCIAS}

1. ARCURI JF, et al. Benefits of interventions for respiratory secretion management in adult palliative care patients: a systematic review. BMC Palliative Care, 2016; 15(1): 74.

2. BAUSEWEIN C, et al. Breathlessness services as a new model of support for patients with respiratory disease. Chronic Respiratory Disease, 2018; 15(1): 48-59.

3. BAUSEWEIN C, SIMON ST. Shortness of Breath and Cough in Patients in Palliative Care. Deutsches Aerzteblatt Online, 2013; 110(33-34): 563-71.

4. BOLAND JW, BOLAND EG. Noisy upper respiratory tract secretions: pharmacological management. BMJ Supportive \& Palliative Care, 2020; 10(3): 304-305.

5. CHAPMAN EJ, et al. Practice review: Evidence-based and effective management of pain in patients with advanced cancer. Palliative Medicine, 2020; 34(4): 444-453.

6. CHIGBO N, et al. Ethics of physiotherapy practice in terminally ill patients in a developing country, Nigeria. Nigerian Journal of Clinical Practice, 2015; 18(7): 40.

7. CREFITO 4 Conselho Regional de fisioterapia e terapia ocupacional da 4a ${ }^{a}$ região, 2015 . Disponível em: https://crefito4.org.br/site/definicao/\#: :text=Defini\%C3\%A7\%C3\%A30\%20de\%20Fisioterapia\%20e\%20\%C3\%81rea s, m\%C3\%A9dia\%20complexidade\%20e\%20alta\%20complexidade. Acesso em: 20 jun. 2020.

8. DIAZ DE TERAN T, et al. Non-invasive ventilation in palliative care: a systematic review. Minerva Medica, 2020; 110(6)

9. EVANGELISTA CB, et al. Cuidados paliativos e espiritualidade: revisao integrativa da literatura. Revista Brasileira de Enfermagem, 2016; 69(3): 591-601. 
10. FARIA L. As práticas do cuidar na oncologia: a experiência da fisioterapia em pacientes com câncer de mama. História, Ciências, Saúde-Manguinhos, 2010; 17(1): 69-87.

11. FERNANDO G, HUGHES S. Team approaches in palliative care: a review of the literature. International Journal of Palliative Nursing, 2019; 25(9): 444-451.

12. FRIKKEL J. et al. Fatigue, barriers to physical activity and predictors for motivation to exercise in advanced Cancer patients. BMC Palliative Care, 2020; 19(1): 43.

13. GIBSON W, et al. Transcutaneous electrical nerve stimulation (TENS) for chronic pain - an overview of Cochrane Reviews. Cochrane Database of Systematic Reviews, 2019; 4(4).

14. GOMES ALZ, OTHERO MB. Cuidados paliativos. Estudos Avançados, dez. 2016; 30(88):155-166.

15. GRADALSKI T. Edema of Advanced Cancer: Prevalence, Etiology, and Conservative Management-A Single Hospice Cross-Sectional Study. Journal of Pain and Symptom Management, 2019; 57(2): 311-318.

16. GRANGER CL. Physiotherapy management of lung cancer. Journal of Physiotherapy, 2016; 62(2): 60-67.

17. GRECO FP, et al. Efeitos dos Exercícios Físicos em Pacientes Submetidos à Quimioterapia Paliativa. Arquivos de Ciências da Saúde, 2019; 26(2): 146.

18. KAKO J. et al. Immediate Effect of Fan Therapy in Terminal Cancer With Dyspnea at Rest: A Meta-Analysis. American Journal of Hospice and Palliative Medicine®, 2020; 37(4): 294-299.

19. KONDO T, et al. Neuromuscular electrical stimulation is feasible in patients with acute heart failure. ESC Heart Failure, 2019; 6(5): 975-982.

20. KUMAR S, SAHA S. Mechanism-based classification of pain for physical therapy management in palliative care: A clinical commentary. Indian Journal of Palliative Care, 2011; 17(1)80.

21. MATILDE INE, et al. Bronchial hygiene techniques in patients on mechanical ventilation: what are used and why? Einstein (São Paulo), 2018; 16(1).

22. MCLEOD KE, NORMAN KE. "I've found it's very meaningful work": Perspectives of physiotherapists providing palliative care in Ontario. Physiotherapy Research International, 2020; 25(1).

23. MCLLWAINE M, et al. Positive expiratory pressure physiotherapy for airway clearance in people with cystic fibrosis. Cochrane Database of Systematic Reviews, 2019.

24. MUSTIAN KM, et al. Comparison of Pharmaceutical, Psychological, and Exercise Treatments for Cancer-Related Fatigue: A Meta-analysis. JAMA Oncology, 2017; 3(7): 961.

25. NAKANO J, et al. Effects of transcutaneous electrical nerve stimulation on physical symptoms in advanced cancer patients receiving palliative care: International Journal of Rehabilitation Research, 2020; 43(1): 62-68.

26. NASCIMENTO DM, et al. Experiência em cuidados paliativos à criança portadora de leucemia: a visão dos profissionais. Ciência \& Saúde Coletiva, 2013; 18(9), 2721-272.

27. OLIVEIRA T, et al. Fisioterapia em cuidados paliativos no contexto da atenção primária à saúde: ensaio teórico. Cadernos Saúde Coletiva, 2019; 27(4): 427-431.

28. OLSSON MÖLLER U, et al.Bridging gaps in everyday life-a free-listing approach to explore the variety of activities performed by physiotherapists in specialized palliative care. BMC Palliative Care, 2018; 17(1): 20.

29. POORT H, et al. Cognitive behavioral therapy or graded exercise therapy compared with usual care for severe fatigue in patients with advanced cancer during treatment: a randomized controlled trial. Annals of Oncology, 2020; 31(1): 115-122.

30. PYSZORA A, et al. Physiotherapy programme reduces fatigue in patients with advanced cancer receiving palliative care: randomized controlled trial. Supportive Care in Cancer, 2017; 25(9): 2899-2908.

31. RIBEIRO O, et al. Nursing professional practice models:an integrative literature review. Revista de Enfermagem Referência, 2016; $\operatorname{serlV}(10): 125-134$.

32. SHOLJAKOVA M, et al. Pain Relief as an Integral Part of the Palliative Care. Open Access Macedonian Journal of Medical Sciences, 2018; 6(4):739-741.

33. SIEMENS W, et al. Transcutaneous electrical nerve stimulation for advanced cancer pain inpatients in specialist palliative care a blinded, randomized, sham-controlled pilot cross-over trial. Supportive Care in Cancer, 2020; 28(11): 5323-5333.

34. SILVA LFA, et al. Conflitos bioéticos: atendimento fisioterapêutico domiciliar a pacientes em condição de terminalidade. Revista Bioética, 2017; 25(1): 148-157.

35. SILVA NVC. A atuação do enfermeiro em cuidados paliativos na unidade de terapia intensiva, MG. Monografia (Especialização em Enfermagem em Terapia Intensiva), Universidade Federal de Minas Gerais, Belo Horizonte, 2019; 23p.

36. The 2015 Quality of Death index: ranking palliative care across the world. London: Economist Intelligence Unit, 2015. Disponível https://www.virtualhospice.ca/Assets/2015\%20Quality\%20of\%20Death\%20Index 20151013163458.pdf. Acesso em: 20 jun. 2020.

37. TORRAS JJ i, PORTA-SALES J. El día a día en cuidados paliativos. ¿Prescribiría usted oxigenoterapia domiciliaria a un paciente con disnea sin hipoxemia? Medicina Paliativa, 2017; 24(2): 96-98.

38. WILSON CM, et al. The Role of Physical Therapists Within Hospice and Palliative Care in the United States and Canada. American Journal of Hospice and Palliative Medicine $\AA_{1}, 2017 ; 34(1)$ : 34-41.

39. WITTRY SA, et al. The Value of Rehabilitation Medicine for Patients Receiving Palliative Care. American Journal of Hospice and Palliative Medicine $\AA$, 2018; 35(6): 889-896.

40. ZIANI MM, et al. Efeitos da terapia manual sobre a dor em mulheres com fibromialgia: uma revisão de literatura. Ciência \& Saúde, 2017; 10(1): 48-23. 